\title{
Gut Microbial Diversity in Rotenone Induced and Transgenically Created PD (Parkinson Disease) Flies of Drosophila melanogaster
}

\author{
Ali Hatami Dehnow, Mysore Siddaiah Krishna* \\ Drosophila Stock Centre, Department of Studies in Zoology, University of Mysore, India
}

Received October 27, 2019; Revised December 14, 2019; Accepted December 25, 2019

Copyright $\bigcirc 2020$ by authors, all rights reserved. Authors agree that this article remains permanently open access under the terms of the Creative Commons Attribution License 4.0 International License

\begin{abstract}
In the present study of gut microbial diversity in control and experimental treated $D$. melanogaster flies showed that there were two species belong to Acetobacter species group namely Acetobacter pomorum and Acetobacter tropicalis and three species belong to Lactobacillus species namely Lactobacillus brevis, Lactobacillus fructivorans and Lactobacillus plantarum. Further analysis revealed that the relative abundance of each of the above microbial species varies in control and experimental flies. The density of lactobacillus species such as L. brevis, L. fructivorans and L. plantarum were found to be lowest in control and creatine supplemented flies. While these species were highest in rotenone induced and transgenically created PD flies. Further A. pomorum and A. tropicalis were found to be lowest abundance in control and creatine treated flies whereas they were found to be greater abundance in rotenone induced and transgenically created PD flies of $D$. melanogaster. Thus, these studies suggest that significant influence of host diet related changes in gut microbial density in Drosophila.
\end{abstract}

Keywords Parkinson's Disease, Microbiota, 16S rRNA, Pyro Sequencing, D.melanogaster Transgenic Flies

\section{Introduction}

The term microbiome of an organism comprises of bacteria, archaeabacteria, fungi, protozoa and viruses. Nowadays the role of microbiota on human physiology has received much attention to understand its effect on health and variations on gut microbe have been linked to numerous human illnesses including neurodegenerative diseases [1]. Realizing this importance in 2007 the national institutes of health (NIH) launched the human microbiome project to identifying and understand the role of microbial species in human health and diseases [1]. Studies of such analysis have revealed that total microbiome present in an organism was more than 100 times the number of genes found in the human genome [2-3].

Changes in the intestine due to gut microbe can influence brain function [4]. Studies on gut brain axis have shown that it is a bidirectional communication system that uses neural, endocrine and immunological signals. In such an axis the neural information is thought to travel along vagal or spinal innervation between the CNS and the gut [4]. The changes in gut microbial diversity could send the signal directly through CNS and indirectly through the synthesis of neurotransmitters or neurochemical-like precursors. Example GABA synthesis by lactobacillus and bifidobacterium species; noradrenaline by Escherichia, bacillus and saccharomyces species; serotonin by candida, streptococcus, Escherichia and enterococcus species; dopamine by bacillus species and ultimately, acetylcholine by lactobacillus [5].

Studies on human PD had shown changes in the microbial resident population had its effect on its metabolites when comparing the human microbiome. Studies carried out on healthy control versus PD patient on human have found significant dysbiosis in PD patient which is correlated to the changes in the relative abundance of gut microbiota. The changes may include appearance and disappearance of particular microbial species and changes in their relative abundance [6-7]. Studies on animal models of PD have shown a link between microbial dysbiosis and neurodegeneration [8]. Such studies revealed that healthy intestinal development requires microbiota colonization and robust antibiotic treatment can also influence the host physiology [9-10]. Studies using pharmacologically -induced PD models 
have shown exposure to rotenone showed variations in abundance reported such as increases in Bifidobacterium and Lactobacillus genus and a decrease in Prevotellaceae after rotenone treatment or the increase in Enterobacteriaceae in MPTP -treated mice were similar to what has been observed on human PD cases [6-7,10-15]. Further these studies have also showed changes in the intestinal phenotype such as gut inflammation and increased intestinal permeability leading to constipation suggesting changes in the gut microbial diversity directly or indirectly was responsible for these changes [9, 16-17].

Drosophila becomes a good animal model to understand the microbial diversity due to its similarity in neuroendocrine architecture with that of higher mammals especially humans [18-19]. Further it is a simple model system to understand the host microbiota relationship and organismal health because of its short life cycle and human-like metabolic traits [18-20]. Studies on microbial diversity in Drosophila have shown that fly has under different situation have used the $16 \mathrm{~s}$ amplicon simple bacterial colonies comprising of four species Lactobacillaceae, Acetobactereace, Enterobactereaceae and Enterococcaceae. Despite variation in the density and composition of these species Lactobacillus and Acetobacter are more common [21-22]. Gut microbial diversity in Drosophila have shown variation in abundance of certain microbial species do occur across various strains and also immune activity. Such studies revealed that maintenance of innate immune homeostasis is associated with the suppression of pathogenic bacteria. Further, as the age increased changes in the innate immune homeostasis can also related to changes in the microbiota [23-25]. Further using germfree flies showed decreased insulin signaling which is promoted by commensal gut bacteria i.e. Lactobacillus Plantarum and Acetobacter Pomorum. Thus, these studies suggest Drosophila forms a very good model to understand changes in health of an organism due to changes associated with its gut microbial diversity. Therefore, present study has been undertaken to understand gut microbial diversity in rotenone induced and transgenically created PD flies of Drosophila.

\section{Materials and Methods}

Details procedure used to establish and maintenance of control and experimental flies [Creatine treated, rotenone treated (rotenone-induced PD flies), co-exposure of rotenone and creatine, transgenic created PD flies] of D.melanogaster were presented in our earlier paper [26]. These flies were subjected to gut microbial analysis.

\subsection{Collection of Gut Microbe and Isolation of DNA}

Midguts of control and experimental flies were removed by using $70 \%$ ethanol. Twenty midguts per experimental flies were isolated to extract DNA using QIA amp DNA mini kit (Qiagen, 51304). Midguts were externally sterilized with $70 \%$ ethanol and using homogenized in $180 \mu \mathrm{L}$ ATL buffer, containing $0.5 \%$ reagent DX for foam minimization using an electric pestle (Kimble $^{\mathrm{TM}}$ Kontes $^{\mathrm{TM}}$ Pellet Pestle, 749540-0000). For additional lysis, $20 \mu \mathrm{L}$ proteinase $\mathrm{K}$ SOLUTION was added to samples and incubated for $30 \mathrm{~min}$ at $56^{\circ} \mathrm{C}$ with shaking at $650 \mathrm{rpm}$. The samples were further lysed by homogenization using glass beads $(425-600 \mu \mathrm{m}$, sigma Aldrich, G8772-100G) in a fast prep FP120 machine (Bio 101 Savant) and afterward incubated for another $60 \mathrm{~min}$ at $56^{\circ} \mathrm{C}$. For RNA digestion, RNase A was added (Qiagen, 19101) and incubated the samples for $2 \mathrm{~min}$ at room temperature. After cool down, $200 \mu \mathrm{L}$ of ethanol was added and the samples were transferred to the spin column as per manufacture instructions the washing and elution steps were performed. The samples were afterward further concentrated by sodium acetate precipitation.

\subsection{Pyro Sequencing of $16 \mathrm{~s}$ rRNA for Identification of Bacterial Species}

Axon-specific 16S rRNA gene primers were used to identify major gut microbial diversity of D. melanogaster such as Acetobacter pomorum, Acetobacter tropicalis, Lactobacillus brevis, Lactobacillus fructivorans and Lactobacillus plantarum using primer3 software and unique regions identified from alignments of full $16 \mathrm{~S}$ rRNA gene sequences. Preliminary experiments confirmed that the primers generated to detectable cross-amplification between species. PCRs were performed as above with $65^{\circ} \mathrm{C}$ annealing temperature and 35 cycles. PCR products were separated by gel electrophoresis using 1\% agarose gel and visualized with SYBR (Invitrogen), and their identities were confirmed by Sanger sequencing.

\subsection{Measurement of Bacterial Loads}

Quantification of gut microbe found in gut of control and experimental flies of D. melanogaster. MRSagar was used for quantifying all microbes except for the strains, which were Acetobacter pomorum quantified on mannitol plates. To measure microbe growth guts were placed on either MRS or mannitol agar plates. Viable bacterial load was calculated on the basis of colony forming units (CFU's) a colony -forming unit is a unit/mi (used to estimate the number of viable bacteria in a sample). Viable is defined as the ability to multiply via binary fission under the controlled condition. Counting with colony forming units requires culturing the microbes and counting only viable cells, in contrast with microscopic examination. Abundance is calculated using colony forming units' which were expressed using logarithmic notation. 


\section{Statistical Analysis}

One-way ANOVA followed by Tukey's post hoc test carried out on the above data showed significant variation in gut microbial diversity between control and experimental flies in all the gut microbial species identified.

\section{Results and Discussion}

Most compelling studies on gut microbial diversity have shown that host diet is an important environmental factor known to affect the gut microbial diversity [6, 27-28]. In the present study two compounds such as rotenone, a known antioxidant inducer and in chronic doses it is known to produce Parkinson disease in D.melanogaster [26, 29]. Creatine, on the other hand, known potent antioxidant $[26,29]$. In the present study, effect of rotenone and creatine supplementation on gut microbial diversity of D.melanogaster was made prior to the experiment the experimental flies were cultured in a wheat cream agar medium and maintained them in the same conditions. In experimental flies rotenone and creatine were treated separately and also in one of experimental flies co exposed with these two compounds. Further PD flies were also created using transgenic flies. The control and above experimental flies were subjected for gut microbial diversity to understand the effect of host diet on physiology in turn its effect on resident microbiota. It was also noticed from the experiment, a total of five microbial species were identified with the help of diagnostic primers listed in the Table 1 and it was also quantified each of the identified species using CFU's (Table 2)

There were two species gut microbe belong to acetobacter species group namely Acetobacter pomorum and Acetobacter tropicalis and three species gut microbe belong to lactobacillus species group namely Lactobacilius brevis, Lactobacillus fructivorans and Lactobacillus plantarum. However, the relative abundance of each species varies in relation to host diet. The density of lactobacillus species such as Lactobacilius brevis, Lactobacillus fructivorans and Lactobacillus plantarum were found to be lowest in control and creatine supplemented flies (Figure 1). On the other hand, they were highest in rotenone induced and transgenically created PD flies.

Table 1. Diagnostic primers used for identification of bacteria.

\begin{tabular}{|c|c|c|c|c|}
\hline \multirow{2}{*}{$\begin{array}{c}\text { Bacterial } \\
\text { species }\end{array}$} & \multicolumn{2}{|l|}{ End point PCR } & \multicolumn{2}{|l|}{ QRT-PCR } \\
\hline & Forward & Reverse & Forward & Reverse \\
\hline $\begin{array}{l}\text { Acetobacter } \\
\text { pomorum }\end{array}$ & $\begin{array}{l}\text { 5'- } \\
\text { TGGGTGGGGGATA } \\
\text { ACACTGGGA-3' }\end{array}$ & $\begin{array}{l}5^{\prime}- \\
\text { AGAGGTCCCTTGC } \\
\text { GGGAAACA-3' }\end{array}$ & $\begin{array}{l}\text { '- } \\
\text { TGTTTCCCGCAAGG } \\
\text { GACCTCT-3' }\end{array}$ & $\begin{array}{l}\text { 5'- } \\
\text { AGAGTGCCCAGCCC } \\
\text { AACCTGA-3' }\end{array}$ \\
\hline $\begin{array}{c}\text { Acetobacter } \\
\text { tropicalis }\end{array}$ & $\begin{array}{l}\text { '- } \\
\text { AGGGCTTGTATGG } \\
\text { GTAGGCT-3' }\end{array}$ & $\begin{array}{l}5^{\prime}- \\
\text { CAGAGTGCAATCC } \\
\text { GAACTGA-3' }\end{array}$ & $\begin{array}{l}5^{\prime}- \\
\text { TAGCTAACGCGATA } \\
\text { AGCACA-3' }\end{array}$ & $\begin{array}{l}5^{\prime}- \\
\text { ACAGCCTACCCATA } \\
\text { CAAGCC-3' }\end{array}$ \\
\hline $\begin{array}{c}\text { Lactobacilius } \\
\text { brevis }\end{array}$ & $\begin{array}{l}\text { 5'- } \\
\text { ACGTAGCCGACCT } \\
\text { GAGAGGGT-3' }\end{array}$ & $\begin{array}{l}\text { 5'- } \\
\text { AGCTTAGCCTCACG } \\
\text { ACTTCGCA-3' }\end{array}$ & - & - \\
\hline $\begin{array}{l}\text { Lactobacillus } \\
\text { fructivorans }\end{array}$ & $\begin{array}{l}\text { '- } \\
\text { TGGATCCGCGGCG } \\
\text { CATTAGC-3' }\end{array}$ & $\begin{array}{l}5^{\prime}- \\
\text { GCCCCCGAAGGGG } \\
\text { ACACCTA-3' }\end{array}$ & $\begin{array}{l}5^{\prime}- \\
\text { AACCTGCCCAGAAG } \\
\text { AAGGGGA-3' }\end{array}$ & $\begin{array}{l}5^{\prime}- \\
\text { GCGCCGCGGATCCA } \\
\text { TCCAAA-3' }\end{array}$ \\
\hline $\begin{array}{l}\text { Lactobacillus } \\
\text { plantarum }\end{array}$ & $\begin{array}{l}'- \\
\text { TCCATGTCCCCGA } \\
\text { AGGGAACG-3' }\end{array}$ & $\begin{array}{l}5^{\prime}- \\
\text { TGGAATGGTCCCG } \\
\text { CGGCGTAT-3' }\end{array}$ & $\begin{array}{l}5^{\prime}- \\
\text { TGTCTCAGTCCCAA } \\
\text { TGTGGCCG-3' }\end{array}$ & $\begin{array}{l}5^{\prime}- \\
\text { GGCTATCACTTTTG } \\
\text { GATGGTCCCGC-3' }\end{array}$ \\
\hline
\end{tabular}

Table 2. Richness and evenness estimation of the microbiota in each of control and experimental flies of D. melanogaster. Diversity estimations were obtained following normalization of OUT'S

\begin{tabular}{lcccccc}
\hline & Control & \multicolumn{6}{c}{ Experimental flies } \\
\cline { 3 - 7 } & flies & $\begin{array}{c}\text { Creatine } \\
\text { supplemented } \\
\text { flies }\end{array}$ & $\begin{array}{c}\text { Rotenone } \\
\text { induced PD }\end{array}$ & $\begin{array}{c}\text { Rotenone } \\
\text { induced PD } \\
\text { +Creatine }\end{array}$ & $\begin{array}{c}\text { Transgenically } \\
\text { Created PD } \\
\text { flies }\end{array}$ & $\begin{array}{c}\text { Transgenically } \\
\text { created PD } \\
\text { flies +Creatine }\end{array}$ \\
OUT'S & 55 & 60 & 63 & 65 & 64 & 62 \\
Chaol & 63 & 64 & 65 & 64 & 67 & 65 \\
Shannon & 2.10 & 3.11 & 3.14 & 3.18 & 3.36 & 3.10 \\
Evenness & 0.72 & 0.74 & 0.78 & 0.79 & 0.80 & 0.74 \\
\hline
\end{tabular}




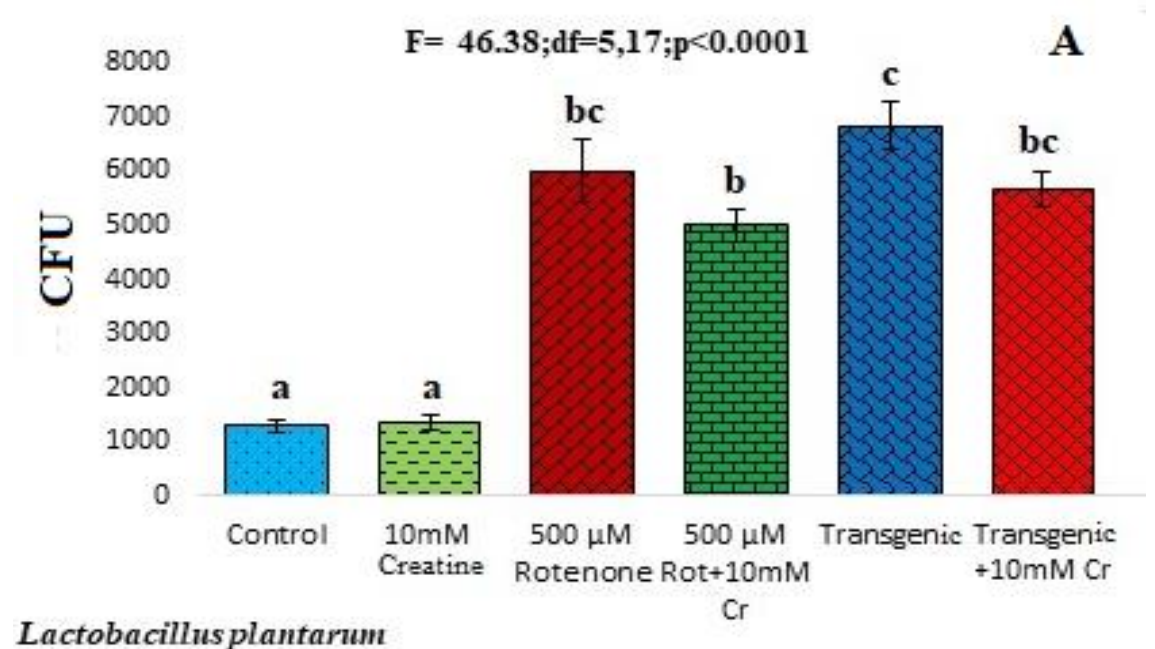

Lactobacillus plantarum
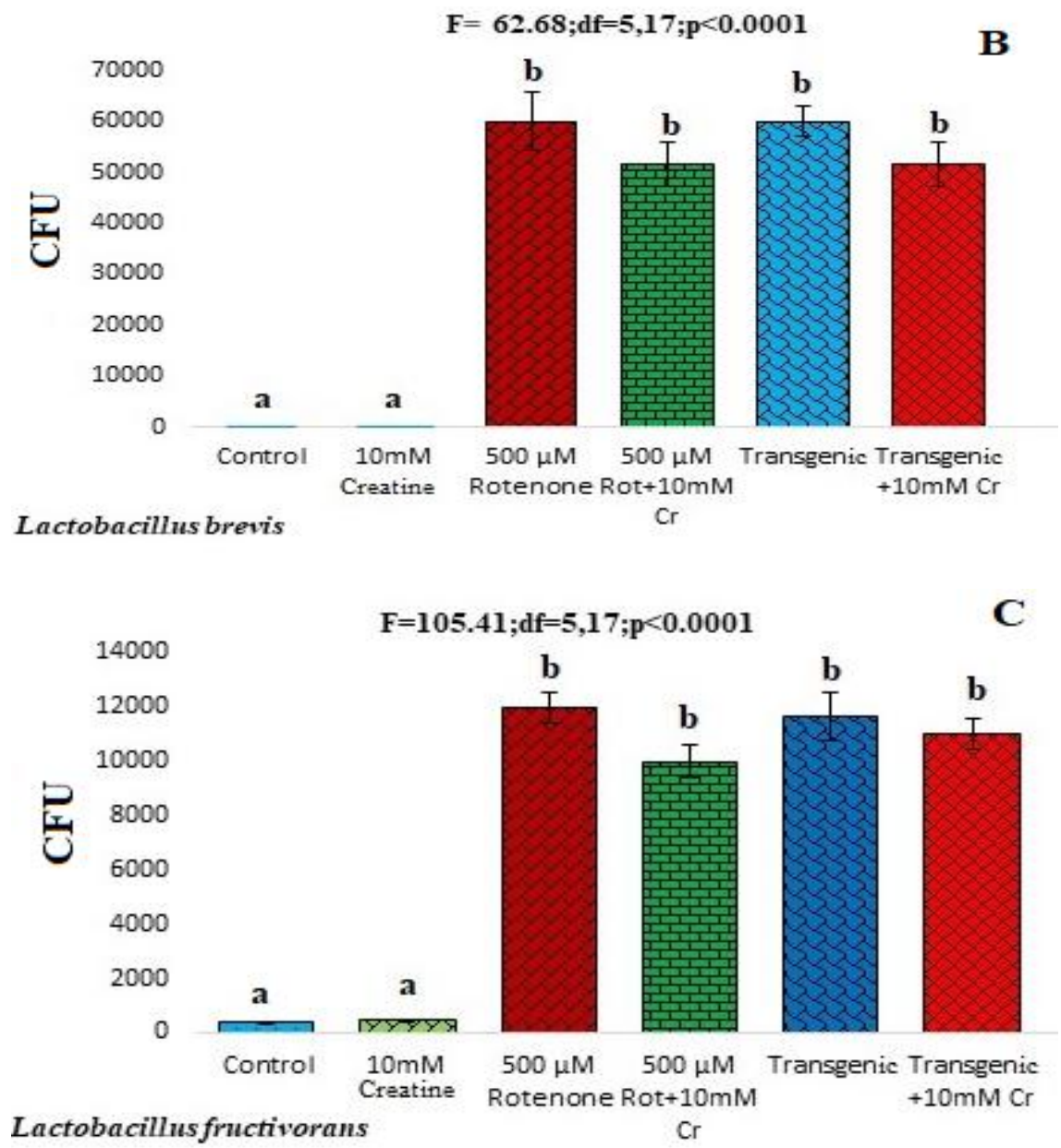

Figure 1. CFU's of Lactobacillus plantarum (A), Lactobacilius brevis (B) and Lactobacillus fructivorans (C) in control and experimental flies of D.melanogaster. Values are mean \pm SE (three replicates). Different letter on the superscript of bar graph indicate significance at $\mathrm{p}<0.05$ level by Tukey's post hoc test 


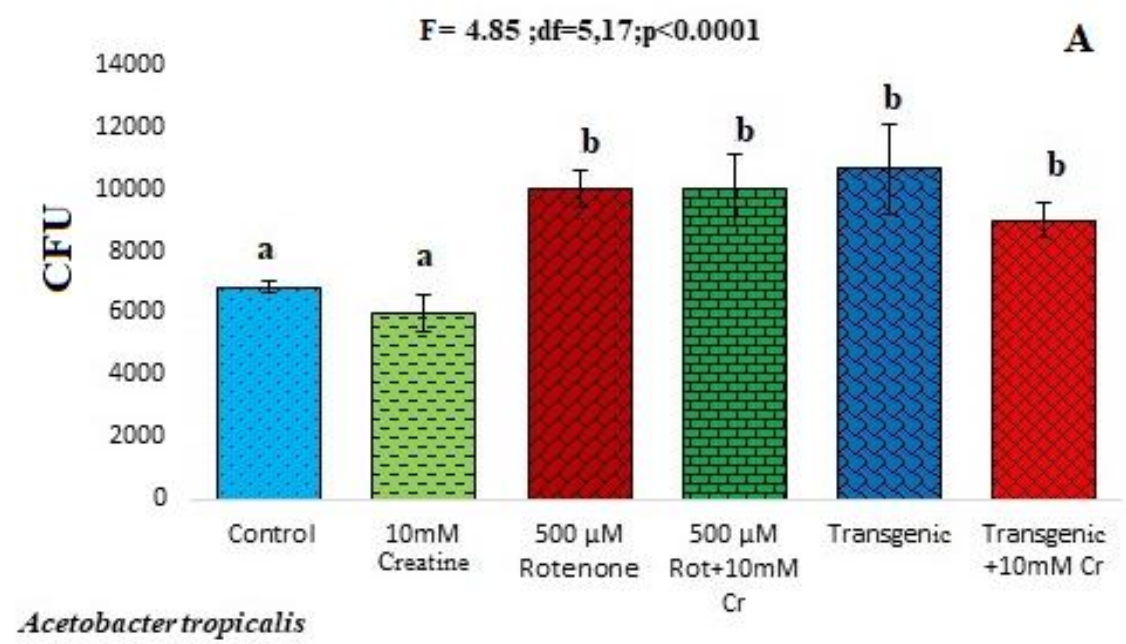

120000

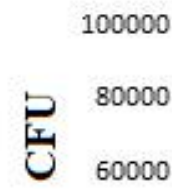

60000

40000

20000

Acetobacter pomorum

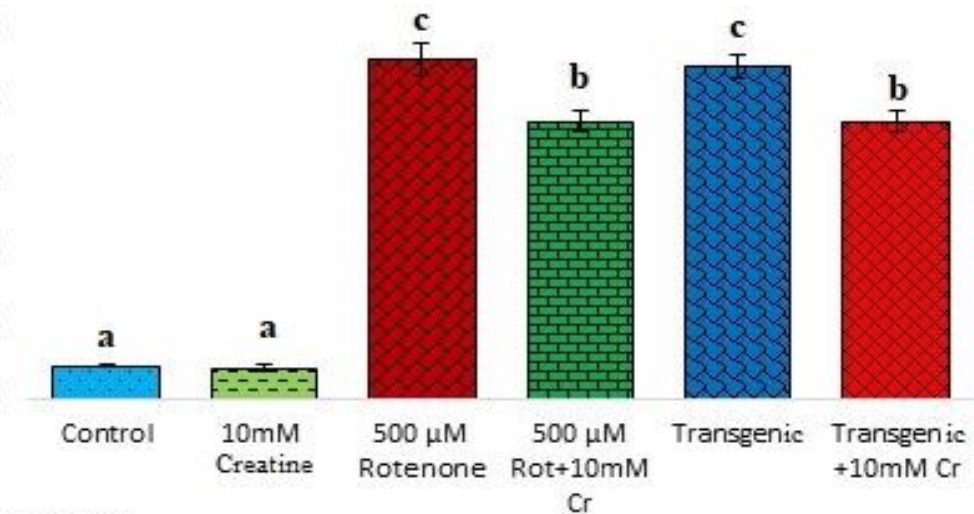

\section{$\mathrm{F}=201.05 ; \mathrm{df}=5,17 ; \mathrm{p}<0.0001$}

B

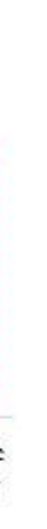

Figure 2. CFU's of Acetobacter tropicalis (A) and Acetobacter pomorum (B) in control and experimental flies of D.melanogaster. Values are mean \pm SE (three replicates). Different letter on the superscript of bar graph indicate significance at $\mathrm{p}<0.05$ level by Tukey's post hoc test

Acetobacter pomorum and Acetobacter tropicalis were found to be lowest abundance in control and creatine treated flies, whereas they were found to be greater abundance in rotenone induced and transgenically created PD flies of D.melanogaster (Figure 2). These results suggest that significant influence of host diet on gut microbial diversity in D.melanogaster. Our result also confirms the earlier studies of host diet related changes in gut microbial in Drosophila [6, 27-28]. This change in gut microbial diversity with diet also shows metabolic changes in the host organism. Earlier studies on gut microbial diversity have recognized that the resident microbiota play important role in animal nutrition [30-33]. This is because the gut microbiota involved in acquisition and allocation of animal nutrients had played key role in shaping the nutritional statues of an animal. Further this gut microbe either consumed ingested nutrient or provide supplementary nutrient to the host thereby they can alert feeding and nutrient assimilation rate.

In the present study, toxic effects of rotenone on microbial diversity in D.melanogaster and potential benefit of creatine supplement to rotenone induced PD and transgenically created PD on gut microbial diversity has been studied. It was noticed from the Figure 1 that species of lactobacillus showed diversity in relation to rotenone treatment. Further the density of lactobacillus species was significantly greater in rotenone and transgenically created PD flies. The density of lactobacillus brevis and Lactobacillus fructivorans were found to be greater (Figure 1). Further creatine supplementation to rotenone induced and transgenically created PD flies had influence in the gut microbiota which caused a moderate reverse of increased bacterial species. This clearly suggests that host diet has significant effect on gut microbial diversity in D.melanogaster. Further in the present study the reads obtained by pyro sequencing each sample were assigned to their respective OTUs and then analyzed for microbiota richness and evenness through determination of their respective indices suggesting that gut microbial diversity varied with host 
diet (Table 2). One-way ANOVA followed by Tukey's post hoc test applied on alone data showed significant variation in gut microbial diversity of lactobacillus and acetobacter species in control and experimental flies of $D$. melanogaster (Figure 1 and Figure 2). Further the observed results can be explained that gut microbial diversity in the present study could be attributed to deleterious effect of rotenone treatment on host physiology. Whereas the creatine supplement had either beneficial (promote host performance) or no desirable effect. The result obtained in the present study was attributed to two folds, host and microbiota do not compete for dietary nutrients which could be an indication of having a lower density of gut microbe, which suggests that the various diet derived nutrients are either not utilized by both host and microbiota or they are in sufficient abundance that their consumption by microbiota does not limit host performance. Further the treatment of rotenone in diet had effect on the gut microbiota which in turn had effect on drosophila performance on diet of low or unbalanced nutrient content, suggesting that the association has a nutritional basis. It was also noticed that the processes contributing to interaction between the microbiota and host metabolism are likely multiple and interactive. It was also suggested that the host signaling pathways regulating the metabolism of males and females may respond differently to microbial products and their absence, and the metabolic traits of the microbiota may be influenced by many metabolic and other physiological differences between the sexes, especially the nutritional demands in females for egg production. Thus these studies suggested that rotenone treatment and creatine supplement had significant influence on gut microbial diversity in D.melanogaster. Tukey's post hoc test showed that control and creatine treated flies had significantly lower number of CFU's in Acetobacter pomorum, Acetobacter tropicalis, Lactobacillus brevis, Lactobacillus fructivorans and Lactobacillus plantarum whereas rotenone induced, transgenically created $\mathrm{PD}$, rotenone induced + creatine and transgenically created PD +creatine had significantly greater number of CFU's in Acetobacter pomorum, Acetobacter tropicalis, Lactobacillus brevis, Lactobacillus fructivorans and Lactobacillus plantarum.

\section{Acknowledgments}

The authors are grateful to the Chairman, Department of Studies in Zoology, University of Mysore, and the Drosophila Stock Centre, the University of Mysore for providing facilities to carry out this work. The authors also thank UGCSAP-CAS-PHASE-1 for financial help.

\section{REFERENCES}

[1] Turnbaugh, P.J., Ley, R.E., Hamady, M., Fraser Liggett, C.M., Knight, R. and Gordon, J.I. (2007) The Human Microbiome Project. Nature. 449: 804-810.

[2] Qin, J., Li, R., Raes, J., Arumugam, M., Burgdorf, K.S., Manichanh, C., Nielsen, T., Pons, N., Levenez, F. and Yamada, T. (2010) A human gut microbial gene catalogue established by metagenomic sequencing. Nature. 464: 59-65.

[3] Qin, R., Fuchs, S. and Milo, R. (2016) Are We Really Vastly Outnumbered? Revisiting the Ratio of Bacterial to Host Cells in Humans. Cell. 164: 337-340.

[4] Erny, D., Hrabe de Angelis, A.L., Jaitin, D., Wieghofer, P., Staszewski, O., David, E., Keren-Shaul, H., Mahlakoiv, T., Jakobshagen, K. and Buch, T. (2015) Host microbiota constantly control maturation and function of microglia in the CNS. Nat. Neuro. Sci. 18: 965-977.

[5] Wall, R., Cryan, J.F., Ross, R.P., Fitzgerald, G.F., Dinan, T.G., Stanton, C., Lyte, M. and Cryan, J.F.(2014) Bacterial Neuroactive Compounds Produced by Psychobiotics. In Microbial Endocrinology: The Microbiota-Gut-Brain Axis in Health and Disease. Springer. 14: 221-239.

[6] Hasegawa, S., Goto, S., Tsuji, H., Okuno, T., Asahara, T., Nomoto, K., Shibata, A., Fujisawa, Y., Minato, T. and Okamoto, A. (2015) Intestinal Dysbiosis and Lowered Serum Lipopolysaccharide-Binding Protein in Parkinson's disease. Plos One. 10:142-164.

[7] Petrov, V.A., Saltykova, I.V., Zhukova, I.A., Alifirova, V.M., Zhukova, N.G., Dorofeeva, Y.B., Tyakht, A.V., Kovarsky, B.A., Alekseev, D.G. and Kostryukova, E.S.(2017) Analysis of Gut Microbiota in Patients with Parkinson's disease. Bul. Exp. Biol. Med.162: 734-737.

[8] Schapira, A.H.V., Chaudhuri, K.R. and Jenner, P. (2017) Non-motor features of Parkinson disease. Nat. Rev. Neurosci. 18: 435-450.

[9] Gerhardt, S. and Mohajeri, M.(2018) Changes of Colonic Bacterial Composition in Parkinson's disease and Other Neurodegenerative Diseases. Nutrients. 10: 708-716.

[10] Peng, C., Gathagan, R.J., Covell, D.J., Medellin, C., Stieber, A., Robinson, J.L., Zhang, B., Pitkin, R.M., Olufemi, M.F. and Luk, K.C. (2018) Cellular milieu imparts distinct pathological $\alpha$-synuclein strains in $\alpha$-synuclein opathies. Nature. 557: 558-563.

[11] Leclercq, S., Mian, F.M., Stanisz, A.M., Bindels, L.B., Cambier, E., Ben-Amram, H., Koren, O., Forsythe, P. and Bienenstock, J.(2017) Low-dose penicillin in early life induces long-term changes in murine gut microbiota, brain cytokines and behavior. Nat. Commun. 8:150-162.

[12] Johnson, M.E., Stringer, A. and Bobrovskaya, L.(2018) Rotenone induces gastrointestinal pathology and microbiota alterations in a rat model of Parkinson's disease. Neuro Toxicology.65: 174-185.

[13] Dodiya, H.B., Forsyth, C.B., Voigt, R.M., Engen, P.A., Patel, J., Shaikh, M., Green, S.J., Naqib, A., Roy, A. and Kordower, J.H.(2018) Chronic stress-induced gut dysfunction exacerbates Parkinson's disease phenotype and pathology in a rotenone-induced mouse model of Parkinson's disease. Neurobiol. 39:1984-1995.

[14] Lai, F., Jiang, R., Xie, W., Liu, X., Tang, Y., Xiao, H., Gao, 
J., Jia, Y. and Bai, Q.(2018) Intestinal Pathology and Gut Microbiota Alterations in a Methyl-4-phenyl -1,2,3,6- tetra hydro pyridine (MPTP) Mouse Model of Parkinson's Disease. Neurochem.43: 1986-1999.

[15] Heintz-Buschart, A., Pandey, U., Wicke, T., Sixel-Döring, F., Janzen, A., Sittig-Wiegand, E., Trenkwalder, C., Oertel, W.H., Mollenhauer, B. and Wilmes, P.(2018) The nasal and gut microbiome in Parkinson's disease and idiopathic rapid eye movement sleep behavior disorder: Nose and Gut Microbiome in PD and iRBD. Mov. Disord.33: 88-98.

[16] Choi, J.G., Kim, N., Ju, I.G., Eo, H., Lim, S.M., Jang, S.E., Kim, D.H. and Oh, M.S.(2018) Oral administration of Proteus mirabilis damages dopaminergic neurons and motor functions in mice. Sci. Rep. 8: 657-670.

[17] Pellegrini, C., Fornai, M., Colucci, R., Tirotta, E., Blandini, F., Levandis, G., Cerri, S., Segnani, C., Ippolito, C. and Bernardini, N.(2016) Alteration of colonic excitatory tachykininergic motility and enteric inflammation following dopaminergic nigrostriatal neurodegeneration. J. Neuroinflam. 13: 743-755

[18] Erkosar, B., Storelli, G., Defaye, A. and Leulier, F.(2013) Host- intestinal microbiota mutualism:"learning on the fly". Cell host microbe. 13: 8-14

[19] Wong, E.V., Cao, W., Vörös, J., Merchant, M., Modis, Y., Hackney, D.D., Montpetit, B., De La. and Cruz, E.M.(2016) Release Limits the Intrinsic and RNA-Stimulated ATPase Cycles of DEAD-Box Protein 5 (Dbp5). J. Mol. Biol. 428: 492-508.

[20] Erkosar, B. and Leulier, F. (2014) Transient adult microbiota, gut homeostasis and longevity: Novel insights from the Drosophila model. FEBS Letters.588: $4250-4257$.

[21] Storelli, G., Defaye, A., Erkosar, B., Hols, P., Royet, J., and Leulier, F.(2011) Lactobacillus plantarum promotes Drosophila systemic growth by modulating hormonal signals through TOR-dependent nutrient sensing. Cell. Metab. 14(3): 403-414.

[22] Wong, J.W., Ho, S.Y. and Hogg, P. J.(2011) Disulfide bond acquisition through eukaryotic protein evolution. Mol, Biol, Evol. 28(1): 327-334.

[23] Lhocine, N., Ribeiro, P.S., Buchon, N., Wepf, A., Wilson, R., Tenev, T., Lemaitre, B., Gstaiger, M., Meier, P. and Leulier, F.(2008) PIMS modulates immune tolerance by negatively regulating Drosophila innate immune signaling. Cell. Host. Microbe. 4(2): 147-158.

[24] Ren, C., Webster, P., Finkel, S.E. and Tower, J.(2007) Increased internal and external bacterial load during Drosophila aging without life-span trade-off. Cell. Metab. 6(2), 144-152.

[25] Broderick, N.A and Lemaitre, B.(2012) Gut-associated microbes of Drosophila melanogaster. Gut. Microbes. 3(4) 307-321.

[26] Ali, H.D. and Krishna, M.S.(2019) Creatine Supplement Effect Similar in Rotenone Induced and Transgenic PD Flies in Drosophila melanogaster. J. Pharm. Chem. Bio. Sci. 7: 84-93.

[27] Hill-Burns, E.M., Debelius, J.W., Morton, J.T., Wissemann,
W.T., Lewis, M.R., Wallen, Z.D., Peddada, S.D., Factor, S.A., Molho, E. and Zabetian, C.P.(2017) Parkinson's disease and Parkinson's disease medications have distinct signatures of the gut microbiome: PD, Medications, and Gut Microbiome. Mov. Disord.32: 739-749.

[28] Scheperjans, F., Aho, V., Pereira, P.A.B., Koskinen, K., Paulin, L., Pekkonen, E., Haapaniemi, E., Kaakkola, S., Eerola-Rautio, J. and Pohja, M.(2015) Gut microbiota are related to Parkinson's disease and clinical phenotype: Gut Microbiota in Parkinson's disease. Mov. Disord. 30: 350-358.

[29] Ravikumar, H. and Saraf, R.R.(2010) Attenuation of Rotenone-Induced Mitochondrial Oxidative Damage and Neurotoxicty in Drosophila melanogaster Supplemented with Creatine. Neurochem Res. 35: 1402-1412.

[30] Le Chatelier, E., Nielsen, T., Qin, J., Prifti, E., Hildebrand, F., Falony, G., Almeida, M., Arumugam, M., Batto, J.M. and Kennedy, S.(2013) Richness of human gut microbiome correlates with metabolic markers. Nature. 500: 541-546.

[31] Ursell, L.K., Clemente, J.C., Rideout, J.R., Gevers, D., Caporaso, J.G. and Knight, R. (2012) The interpersonal and intrapersonal diversity of human-associated microbiota in key body sites. J. Allergy. Clin. Immun. 129: 1204-1208.

[32] Lloyd-Price, J., Mahurkar, A., Rahnavard, G., Crabtree, J., Orvis, J., Hall, A.B., Brady, A., Creasy, H.H., McCracken, C. and Giglio, M.G.(2017) Strains, functions and dynamics in the expanded Human Microbiome Project. Nature. 550: 61-66.

[33] Swann, J.R., Want, E.J., Geier, F.M., Spagou, K., Wilson, I.D., Sidaway, J.E., Nicholson, J.K. and Holmes, E.(2011) Systemic gut microbial modulation of bile acid metabolism in host tissue compartments. Proc. Natl. Acad. Sci.108: 4523-4530. 